\title{
A Model for Compression-Weakening Materials and the Elastic Fields due to Contractile Cells
}

\author{
Phoebus Rosakis \\ Department of Theoretical and Applied Mathematics \\ University of Crete, Heraklion 70013, Greece \\ rosakis@uoc.gr \\ Jacob Notbohm \\ Department of Engineering Physics, University of Wisconsin \\ Madison, WI 53706, USA \\ jknotbohm@wisc.edu \\ Guruswami Ravichandran \\ Division of Engineering and Applied Science \\ California Institute of Technology, Pasadena CA 91125, USA \\ ravi@caltech.edu
}

\begin{abstract}
We construct a homogeneous, nonlinear elastic constitutive law, that models aspects of the mechanical behavior of inhomogeneous fibrin networks. Fibers in such networks buckle when in compression. We model this as a loss of stiffness in compression in the stress-strain relations of the homogeneous constitutive model. Problems that model a contracting biological cell in a finite matrix are solved. It is found that matrix displacements and stresses induced by cell contraction decay slower (with distance from the cell) in a compression weakening material, than linear elasticity would predict. This points toward a mechanism for long-range cell mechanosensing. In contrast, an expanding cell would induce displacements that decay faster than in a linear elastic matrix.
\end{abstract}

\section{Introduction}

Biological cells can sense the mechanical state of the surrounding extracellular matrix, such as stiffness [1], deformations, forces, or stress [2, 3, 4, 5, 6]. This is known as mechanosensing [7]. At the same time, cells actively contract, thereby applying tractions on the extracellular matrix and deform- 


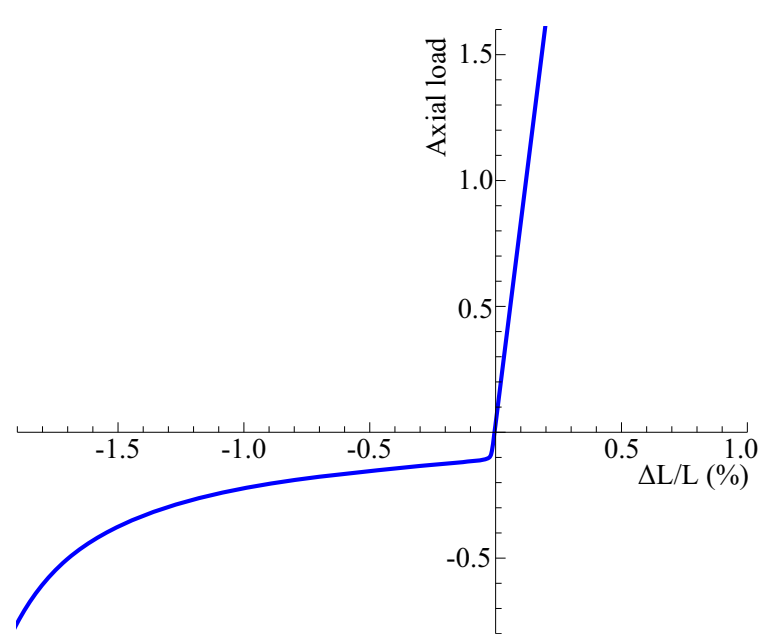

(a)

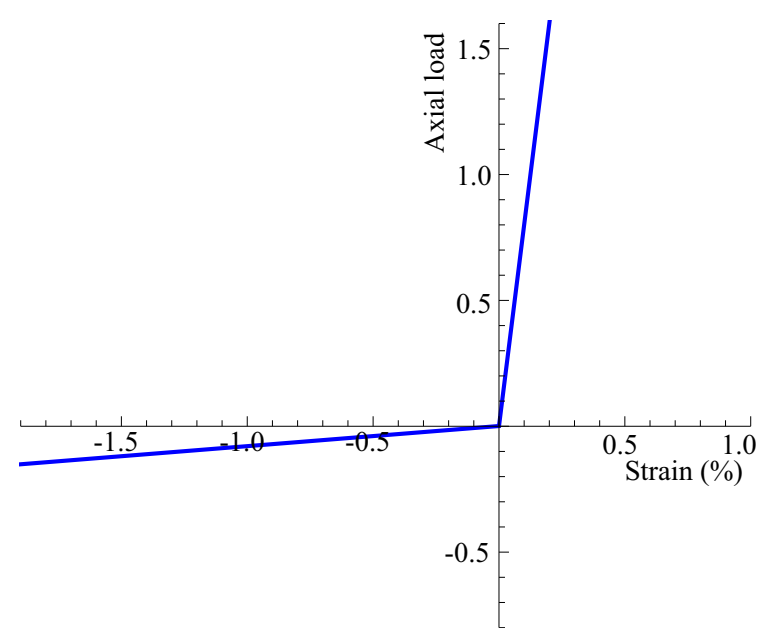

(b)

Figure 1: (a) Typical relation between axial load (vertical axis, arbitrary units) and fractional change in the distance between endpoints (horizontal axis, percent) of an elastic beam that can buckle. (b) One-dimensional piecewise-linear stress-strain curve for a material that weakens in compression. Here $\rho=0.1$. Horizontal axis: strain $\varepsilon$ in percent. Vertical axis: normalized stress $\sigma / \alpha$, where $\alpha$ is a one-dimensional elastic constant.

ing it. The resulting displacement or stress fields can serve as signals to other cells $[3,4,5]$, thus enabling neighboring cells to detect each other $[8,9]$.

Experiments using digital volume correlation with confocal microscopy [10] measured displacements in a 3D fibrin matrix caused by contractile fibroblasts seeded in it $[8,9]$. The fibrin matrix is not a homogeneous material, but rather a random network of slender fibers. The matrix displacements induced by cell contraction were found to decay much slower with distance from the cell than linear elasticity would predict. Thus, contractile cells embedded within a fibrin matrix can detect mechanical fields induced by each other at larger distances, compared to cells in a homogeneous gel matrix that behaves like a linear elastic material, or cells on a linear elastic substrate where displacements decay even faster (exponentially [6]). This observation of long-range cell-cell mechanical communication in 3D agrees with previous experiments that showed a similar effect for cells on a 2D fibrin substrate $[4,11]$. The mechanism for the long-range mechanosensing stems from the mechanical behavior of the fibrous network. It was shown $[8,9]$ that the displacements due to a contracting inclusion in a fiber network decay slower than in a homogeneous linear elastic material, because fibers lose stiffness in compression. The stiffness loss is due to microbuckling, namely buckling of individual fibers in the network that are in compression. See $[12,13,14]$ for various aspects of microbuckling.

We previously developed a finite element fiber network model $[8,9]$ that treated individual fibers as elements whose force-extension curve has smaller slope in compression than in tension, as in 
Fig. 1(b). This is an idealization of the typical relation between axial load and fractional change in the distance between endpoints of an elastic beam that can buckle, shown in Fig. 1(a). One notes the abrupt change of stiffness that occurs at a negative value of the load (the buckling load) in Fig. 1(a). The magnitude of the buckling load depends on the bending stiffness of fibers. For fibrin, the bending stiffness has been found to be nearly two orders of magnitude less than the value predicted by the pure bending model of linear elasticity [15]. Accordingly, the buckling load is essentially taken to vanish in Fig. 1(b). Simulations of our finite element model in 2D [8, 9] with fiber elements obeying the compression weakening stress-strain law of Fig. 1(b) show that matrix displacements induced by a contracting spherical inclusion (representing the cell) decay according to a power law $u \sim r^{-n}$ with distance $r$ from the inclusion center. Values of $n$ depend on the connectivity of the network, but are always in the range $0.2-0.5$, far below the value $n=1$ that $2 \mathrm{D}$ linear elasticity would predict. In $3 \mathrm{D}$, values of $n$ from simulations were in the range $0.6-0.9$, and experiments yielded $n=0.52$, again much less than the linear elastic value $n=2$. Significantly, when the microbuckling elements were replaced by linear elastic ones that do not buckle (same stiffness in compression as in tension) the network simulations yielded values of $n$ close to the linear elastic predictions [9].

These finite element simulations of discrete network models provided strong evidence for the hypothesis formulated by Notbohm [8] and coworkers [9]: microbuckling of fibrin enables long-range cell-induced displacements that facilitate mechanosensing. The purpose of this paper is to provide theoretical support for this conclusion. So far, the evidence comes from experiments and numerical simulations of a discrete network [8,9]. Here we conjecture that a homogeneous solid with lower stiffness in compression than in tension will also exhibit slower decay of displacements/stresses due to a contracting inclusion, than a linear elastic solid. In other words, the dominant factor responsible for the slow displacement decay is loss of stiffness in compression, rather than the discrete character of the fiber network. We show this by first constructing a constitutive model with the requisite properties, then solving some relevant boundary value problems analytically.

Constitutive modeling is described in detail in Section 2. The analysis of relevant boundary value problems is spelled out in Sections 3, 4. Sections 2, 3 and 4 contain considerable technical details and may be omitted at first reading. We briefly present our main results in Section 5 (Results) and discuss their significance in Section 6 (Discussion). 


\section{Constitutive Model}

\subsection{D Constitutive Law}

We now describe a special elastic constitutive law, which loses stiffness in compression in a sense to be made precise. Such a constitutive law cannot be linear, even in the context of small deformations, if it is going to exhibit behavior analogous to that of Fig. 1(b) in more than one dimension. For simplicity we consider small deformations (linearized kinematics). The constitutive law itself is nonlinear; it is isotropic, while the principal stresses are piecewise linear functions of the principal strains (eigenvalues of the infinitesimal strain tensor). This last property allows us to solve some problems of interest analytically.

To begin with, suppose the matrix plus the cell together occupy the whole $2 \mathrm{D}$ space $\mathbb{R}^{2}$ and is composed of linear elastic homogeneous isotropic material (undergoing small deformations, so that the linearized theory of elasticity is used). We thus have a displacement field $\boldsymbol{u}: \mathbb{R}^{2} \rightarrow \mathbb{R}^{2}$. The components of the (infinitesimal) strain tensor are

$$
\boldsymbol{E}=\frac{1}{2}\left(\nabla \boldsymbol{u}+\nabla \boldsymbol{u}^{T}\right), \quad E_{i j}=\frac{1}{2}\left(\frac{\partial u_{i}}{\partial x_{j}}+\frac{\partial u_{j}}{\partial x_{i}}\right)
$$

In the matrix except the cell, the stress tensor is related to the strain tensor by

$$
\boldsymbol{S}=\lambda(\operatorname{tr} \boldsymbol{E}) \mathbf{1}+2 \mu \boldsymbol{E}
$$

Here $\lambda$ and $\mu$ are the Lamé constants and 1 the identity tensor. In components, the above reads ${ }^{1}$

$$
S_{i j}=\lambda E_{k k} \delta_{i j}+2 \mu E_{i j}
$$

The principal stresses $\sigma_{i}$ (the eigenvalues of the stress tensor) are related to the principal strains $\varepsilon_{i}$ (the eigenvalues of the strain tensor) through

$$
\sigma_{i}=C_{i j} \varepsilon_{j}
$$

where

$$
C=\left(\begin{array}{ll}
\alpha & \beta \\
\beta & \alpha
\end{array}\right), \quad \alpha=\lambda+2 \mu, \quad \beta=\lambda,
$$

and $\lambda, \mu$ are the Lamé Moduli (elastic constants; $\mu$ is the shear modulus). In other words, the linear elastic principal stress-strain relations are

$$
\sigma_{1}=\alpha \varepsilon_{1}+\beta \varepsilon_{2}, \quad \sigma_{2}=\beta \varepsilon_{1}+\alpha \varepsilon_{2} .
$$

\footnotetext{
${ }^{1}$ The Einstein summation convention is used: summation over repeated indices is implied, unless indicated otherwise
} 
Positive definiteness of $C$ is equivalent to

$$
\alpha>|\beta|
$$

Our first attempt toward constructing a constitutive law that weakens in compression is to consider piecewise-linear stress-strain relations. Consider the function

$$
Z_{\rho}(x)= \begin{cases}x, & x \geq 0 \\ \rho x, & x<0\end{cases}
$$

where $0 \leq \rho \leq 1$ is the constant compression stiffness ratio. The graph of $Z_{\rho}$ is the curve in Fig. 1(b). Note that the function $Z_{\rho}$ is not linear, but it is positive-homogeneous of degree one, i.e., $Z_{\rho}(\alpha x)=\alpha Z_{\rho}(x)$ for any $\alpha>0$ (and any real $\left.x\right)$.

In $1 \mathrm{D}$, one might replace the linear stress-strain relation $\sigma=\alpha \varepsilon$, where $\alpha$ is a modulus, by the piecewise linear stress-strain relation $\sigma=Z_{\rho}(\alpha \varepsilon)$; see Fig. 1(b). By analogy, in our first attempt, we replace (2.2) with

$$
\begin{aligned}
& \sigma_{1}=Z_{\rho}\left(\alpha \varepsilon_{1}+\beta \varepsilon_{2}\right), \\
& \sigma_{2}=Z_{\rho}\left(\beta \varepsilon_{1}+\alpha \varepsilon_{2}\right) .
\end{aligned}
$$

In effect this multiplies stiffness by $\rho$ whenever the corresponding principal stress is negative (compressive). However, this turns out to be problematic. Suppose the argument of $Z_{\rho}$ in (2.5) is positive and the argument of $Z_{\rho}$ in (2.6) is negative. Then we have $\partial \sigma_{1} / \partial \varepsilon_{2}=\beta$ while $\partial \sigma_{2} / \partial \varepsilon_{1}=\rho \beta$, which means that if $\rho \neq 1$, there is no strain energy density function $W\left(\varepsilon_{1}, \varepsilon_{2}\right)$ such that $\sigma_{i}=\partial W / \partial \varepsilon_{i}$. Thus the stress-strain relations (2.5), (2.6) are not hyperelastic, except in the trivial case of no weakening ( $\rho=1)$ which coincides with linear elasticity. Therefore this model is not satisfactory.

In order to overcome the lack of hyperelasticity just encountered, one might attempt to construct the strain energy function directly. This is somewhat difficult, however, since the change in stiffness is supposed to occur when stresses, not strains, change sign. It is more natural to construct the complementary energy density (a function of stress) $U\left(\sigma_{1}, \sigma_{2}\right)$, with the property that

$$
\frac{\partial U\left(\sigma_{1}, \sigma_{2}\right)}{\partial \sigma_{i}}=\varepsilon_{i} .
$$

Assuming that $W\left(\varepsilon_{1}, \varepsilon_{2}\right)$ is strictly convex and continuously differentiable, the stress-strain relations are invertible to the from $\varepsilon_{i}=\hat{\varepsilon}_{i}\left(\sigma_{1}, \sigma_{2}\right)$ and one has

$$
U\left(\sigma_{1}, \sigma_{2}\right)=\sigma_{i} \varepsilon_{i}-W\left(\varepsilon_{1}, \varepsilon_{2}\right), \quad \varepsilon_{i}=\hat{\varepsilon}_{i}\left(\sigma_{1}, \sigma_{2}\right)
$$

In $1 \mathrm{D}$, one might adopt the stress-strain relation $\sigma=\alpha Z_{\rho}(\varepsilon)$, where $\alpha$ is a modulus. Then since $\sigma=W^{\prime}(\varepsilon)$, we have

$$
W(\varepsilon)=\frac{\alpha}{2} Z_{\sqrt{\rho}}^{2}(\varepsilon) .
$$


Also since the strain-stress relation is $\varepsilon=\alpha^{-1} Z_{\rho^{-1}}(\sigma)$, the complementary energy is

$$
U(\sigma)=\frac{\kappa}{2} Z_{d}^{2}(\sigma), \quad d=1 / \sqrt{\rho}, \quad \kappa=1 / \alpha .
$$

The complementary energy is quadratic in $Z_{d}(\sigma)$, hence piecewise quadratic in $\sigma$, while for linear elasticity $(d=1)$ it would be $\kappa \sigma^{2} / 2$. Now in $2 \mathrm{D}$ for linear elasticity, the complementary energy is

$$
U_{1}\left(\sigma_{1}, \sigma_{2}\right)=\frac{1}{2} K_{i j} \sigma_{i} \sigma_{j}, \quad K=C^{-1} .
$$

Thus one might be tempted to replace $\sigma_{i}$ by $Z_{d}\left(\sigma_{i}\right)$ above and to consider the complementary energy candidate

$$
U_{*}\left(\sigma_{1}, \sigma_{2}\right)=K_{11} Z_{d}^{2}\left(\sigma_{1}\right) / 2+K_{22} Z_{d}^{2}\left(\sigma_{2}\right) / 2+K_{12} Z_{d}\left(\sigma_{1}\right) Z_{d}\left(\sigma_{2}\right) .
$$

The problem with this is that the resulting strain-stress relations (partial derivatives of $U_{*}$ ) are not continuous functions of $\sigma_{i}$. While $Z_{d}^{2}(\sigma)$ is continuously differentiable in $\sigma, Z_{d}(\sigma)$ is not. So while the first two terms above are continuously differentiable, the mixed third term involving $K_{12}$ is not, and (2.9) is not satisfactory. This problem is easily fixed by modifying the third term, and replacing it by the simplest possible coupling between $\sigma_{1}$ and $\sigma_{2}$. We thus choose the complementary energy density for our constitutive model to be

$$
U_{\rho}\left(\sigma_{1}, \sigma_{2}\right)=\frac{1}{2} K_{11} Z_{d}^{2}\left(\sigma_{1}\right)+\frac{1}{2} K_{22} Z_{d}^{2}\left(\sigma_{2}\right)+K_{12} \sigma_{1} \sigma_{2},
$$

where $d=1 / \sqrt{\rho}$. This is once continuously differentiable (but only piecewise twice). Its partial derivative with respect to $\sigma_{i}$, namely $\varepsilon_{i}$, depends on $\sigma_{i}$ (with same index $i$ ) in a piecewise linear fashion, with a change of slope when $\sigma_{i}$ changes sign. Assuming $C$ and hence $K$ to be positive definite, one can show that $U_{\rho}$ is strictly convex. Thus the strain-stress relations are invertible and piecewise linear, and so are the stress-strain relations, while the associated strain energy is strictly convex, piecewise quadratic, and once continuously differentiable. Also, (2.10) coincides with the linear elastic complementary energy (2.8) whenever both $\sigma_{i} \geq 0$ (in the first quadrant of the principal stress plane). The stiffnesses change though whenever one or both of the $\sigma_{i}$ become negative. Thus $U_{\rho}$ coincides with a different quadratic function within each of the four quadrants of the principal stress plane. In particular, noting that $K=C^{-1}$, or

$$
K=\left[K_{i j}\right]=\frac{1}{\alpha^{2}-\beta^{2}}\left(\begin{array}{cc}
\alpha & -\beta \\
-\beta & \alpha
\end{array}\right),
$$

one can write

$$
U_{\rho}\left(\sigma_{1}, \sigma_{2}\right)=\frac{1}{2} \hat{K}_{i j} \sigma_{i} \sigma_{j}
$$


where the matrix $\hat{K}$, apart from $\rho$, also depends on $\sigma_{i}$ in a piecewise constant fashion. Specifically, it depends only on the signs of $\sigma_{i}$, and takes the following four values in the corresponding four quadrants of the principal stress plane (ordered counterclockwise).

$$
\left(\begin{array}{ll}
K_{11} & K_{12} \\
K_{12} & K_{22}
\end{array}\right), \quad\left(\begin{array}{cc}
K_{11} / \rho & K_{12} \\
K_{12} & K_{22}
\end{array}\right), \quad\left(\begin{array}{cc}
K_{11} / \rho & K_{12} \\
K_{12} & K_{22} / \rho
\end{array}\right), \quad\left(\begin{array}{cc}
K_{11} & K_{12} \\
K_{12} & K_{22} / \rho
\end{array}\right) .
$$

The strain-stress relations are easily obtained from (2.10) using (2.7), noting that $K_{11}=K_{22}$ :

$$
\varepsilon_{i}=K_{11} Z_{\rho^{-1}}\left(\sigma_{i}\right)+K_{12} \sigma_{j}, \quad j \neq i
$$

It is possible to construct the strain energy density out of (2.10), (2.12). For a quadratic complementary energy of the form (2.11), the corresponding strain energy is also quadratic:

$$
W_{\rho}\left(\varepsilon_{1}, \varepsilon_{2}\right)=\frac{1}{2} \hat{C}_{i j} \varepsilon_{i} \varepsilon_{j}
$$

where $\hat{C}=\hat{K}^{-1}$. Since $U_{\rho}$ is piecewise quadratic, so is $W_{\rho}$. In particular, $\hat{C}$ is a piecewise constant matrix that takes four values, the inverses of (2.12), in four sectors of the principal strain plane that are the images of the four quadrants of the stress plane under the mapping $\left(\sigma_{1}, \sigma_{2}\right) \mapsto\left(\varepsilon_{1}, \varepsilon_{2}\right)$ defined by the strain-stress relations (2.13). See Fig. 2(a). Switching between these four sectors occurs at points on the uniaxial stress lines; these straight lines are where the principal stresses change signs. See Fig. 2(c). Thus for example in the sector corresponding to $\sigma_{1}>0, \sigma_{2}<0$, the value of $\hat{C}$ in (2.14) is equal to the inverse of the fourth matrix in (2.12):

$$
\hat{C}=\frac{\left(\alpha^{2}-\beta^{2}\right) \rho}{\alpha^{2}-\beta^{2} \rho}\left(\begin{array}{cc}
\alpha / \rho & \beta \\
\beta & \alpha
\end{array}\right), \quad \text { provided } \alpha \varepsilon_{1} / \rho+\beta \varepsilon_{2}>0, \quad \beta \varepsilon_{1}+\alpha \varepsilon_{2}<0 .
$$

The two inequalities above define the sector in the principal strain plane that corresponds to the quadrant $\sigma_{1}>0, \sigma_{2}<0$. Whenever these two inequalities hold, the stress-strain law is arrived at by differentiating (2.14) (with $\hat{C}$ as in (2.15)) with respect to $\varepsilon_{i}$. The result is

$$
\sigma_{1}=h(\rho)\left(\alpha \varepsilon_{1} / \rho+\beta \varepsilon_{2}\right), \quad \sigma_{2}=h(\rho)\left(\beta \varepsilon_{1}+\alpha \varepsilon_{2}\right), \quad \text { where } h(\rho)=\frac{\left(\alpha^{2}-\beta^{2}\right) \rho}{\alpha^{2}-\beta^{2} \rho} .
$$

\subsection{D Constitutive Law}

In 3D (letting Latin indices range over $\{1,2,3\}$ ), the linear elastic isotropic strain energy function in terms of principal strains is

$$
W\left(\varepsilon_{1}, \varepsilon_{2}, \varepsilon_{3}\right)=C_{i j} \varepsilon_{i} \varepsilon_{j}, \quad C=\left(\begin{array}{ccc}
\alpha & \beta & \beta \\
\beta & \alpha & \beta \\
\beta & \beta & \alpha
\end{array}\right)
$$




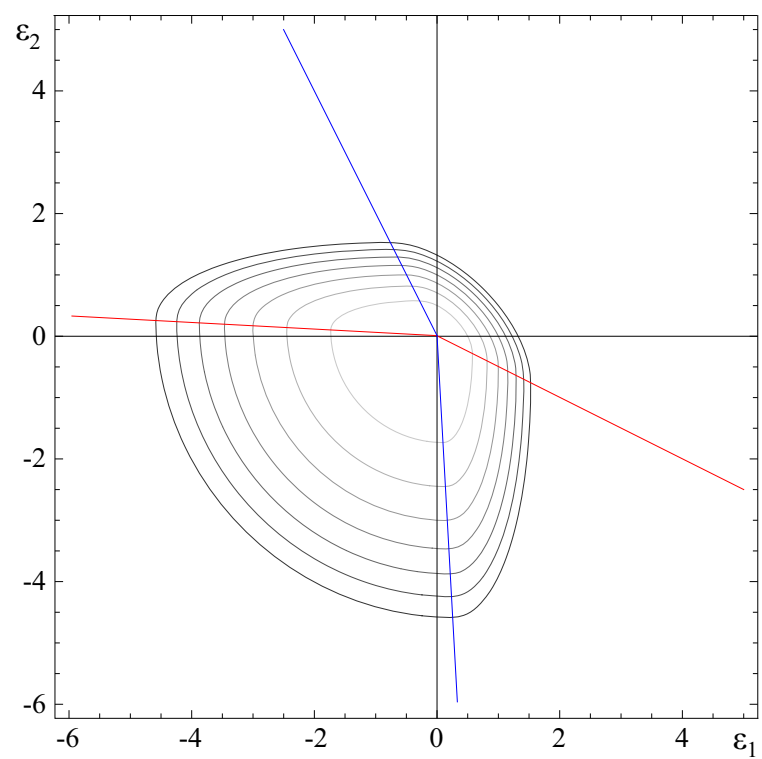

(a)

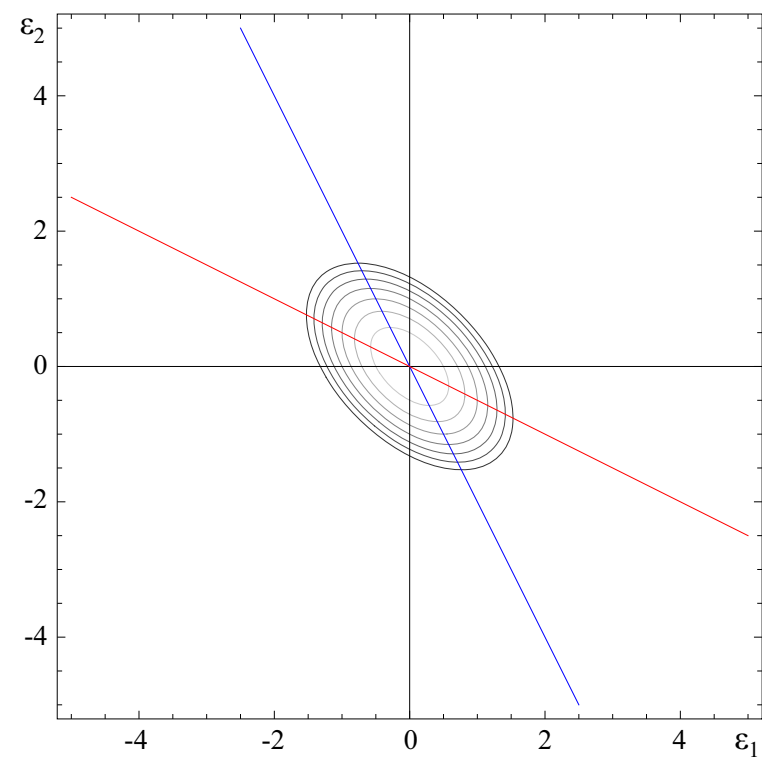

(b)

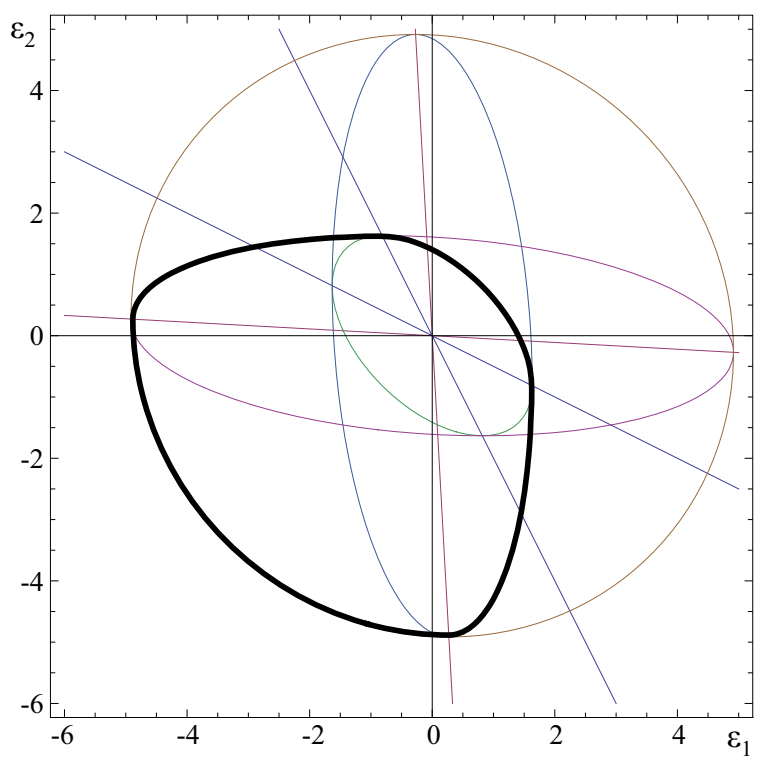

(c)

Figure 2: (a) Level curves of the strain energy density $W_{\rho}$ in the $\left(\varepsilon_{1}, \varepsilon_{2}\right)$ plane. Here $\lambda=2 \mu$ and $\rho=1 / 9$; values of $W_{\rho}$ on the level sets are $10^{-4} \times k \lambda$ for $k=1,2, \ldots, 7$. Values of strain are in percent. The blue and red straight lines separate the plane into four sectors. The strain energy density is equal to a different quadratic function in each sector. The blue line corresponds to uniaxial stress, $\sigma_{1}=0$. The red line corresponds to uniaxial stress, $\sigma_{2}=0$. (b) For comparison, level curves of the corresponding linear elastic energy $(\rho=1)$. Blue and red lines correspond to uniaxial stress. (c) A single level curve of the strain energy density (thick curve). The four ellipses (blue, green, purple and brown) are the level curves of the four quadratic functions that equal the strain energy in different sectors. Switching between these four branches occurs at points on the uniaxial stress lines; these straight lines are where the principal stresses change signs. 
where $\alpha=2 \mu+\lambda, \beta=\lambda$ as before. Positive definiteness of $C$ is equivalent to

$$
\alpha-\beta>0, \quad \alpha+2 \beta>0
$$

(equivalent to $\mu>0,3 \lambda+2 \mu>0$ ). The (principal) stress-strain relations are

$$
\sigma_{i}=C_{i j} \varepsilon_{j}
$$

For the compression-weakening material we construct the complementary energy in analogy to (2.10)

$$
U\left(\sigma_{1}, \sigma_{2}, \sigma_{3}\right)=\frac{1}{2} \sum_{i=1}^{3} K_{i i} Z_{d}^{2}\left(\sigma_{i}\right)+\frac{1}{2} \sum_{i=1}^{3} \sum_{j=1, j \neq i}^{3} K_{i j} \sigma_{i} \sigma_{j}
$$

where

$$
K=\frac{1}{(\alpha-\beta)(\alpha+2 \beta)}\left(\begin{array}{ccc}
\alpha+\beta & -\beta & -\beta \\
-\beta & \alpha+\beta & -\beta \\
-\beta & -\beta & \alpha+\beta
\end{array}\right) .
$$

In particular, this function is continuously differentiable and piecewise quadratic. The second derivatives are piecewise constant and suffer jump discontinuities across the planes $\sigma_{i}=0$ in 3D principal stress space. Thus $U$ equals a quadratic function in each octant. In particular, in the octant

$$
\sigma_{1}>0, \quad \sigma_{2}<0, \quad \sigma_{3}<0
$$

we have that

$$
U\left(\sigma_{1}, \sigma_{2}, \sigma_{3}\right)=\hat{K}_{i j} \sigma_{i} \sigma_{j}, \quad \hat{K}=\frac{1}{(\alpha-\beta)(\alpha+2 \beta)}\left(\begin{array}{ccc}
\alpha+\beta & -\beta & -\beta \\
-\beta & \frac{\alpha+\beta}{\rho} & -\beta \\
-\beta & -\beta & \frac{\alpha+\beta}{\rho}
\end{array}\right) .
$$

In this octant, the strain-stress relations read $\varepsilon_{i}=\hat{K}_{i j} \sigma_{j}$. These are invertible for $0<\rho \leq 1$. The inverse can be found explicitly. The stress-strain relations $\sigma_{i}=\hat{C}_{i j} \varepsilon_{j}$ with $\hat{C}=\hat{K}^{-1}$ ( $\hat{K}$ as above) are valid in the sector of principal strain space which is the image of the octant (2.18).

\subsection{Mechanical Behavior}

The stress-strain relations due to the constitutive law just constructed are as follows. Let $\hat{\sigma}_{i}\left(\varepsilon_{1}, \varepsilon_{2}\right)=$ $\partial W_{\rho}\left(\varepsilon_{1}, \varepsilon_{2}\right) / \partial \varepsilon_{i}$, with $W_{\rho}$ the strain energy density in terms of principal strains from (2.14). Suppose the spectral representation of the strain tensor is ${ }^{2} \boldsymbol{E}=\sum_{i=1}^{2} \varepsilon_{i} \boldsymbol{v}_{i} \otimes \boldsymbol{v}_{i}$, with $\varepsilon_{i}$ the eigenvalues (principal strains) and $\boldsymbol{v}_{i}$ the eigenvectors of $\boldsymbol{E}$. Define the tensor function

$$
\hat{\boldsymbol{S}}(\boldsymbol{E})=\sum_{i=1}^{2} \hat{\sigma}_{i}\left(\varepsilon_{1}, \varepsilon_{2}\right) \boldsymbol{v}_{i} \otimes \boldsymbol{v}_{i}
$$

\footnotetext{
${ }^{2}$ Here for example $\boldsymbol{m} \otimes \boldsymbol{u}$ is the tensor with components $m_{i} u_{j}$.
} 
The tensor stress-strain relation is $\boldsymbol{S}=\hat{\boldsymbol{S}}(\boldsymbol{E})$, so that the principal stresses are given by $\hat{\sigma}_{i}\left(\varepsilon_{1}, \varepsilon_{2}\right)$ in terms of the principal strains, while $\boldsymbol{S}$ has the same eigenvectors $\boldsymbol{v}_{i}$ as $\boldsymbol{E}$. The inverse, strain-stress relation, is given by $\boldsymbol{E}=\hat{\boldsymbol{E}}(\boldsymbol{S})$, where

$$
\hat{\boldsymbol{E}}(\boldsymbol{S})=\sum_{i=1}^{2} \hat{\varepsilon}_{i}\left(\sigma_{1}, \sigma_{2}\right) \boldsymbol{v}_{i} \otimes \boldsymbol{v}_{i}, \quad \boldsymbol{S}=\sum_{i=1}^{2} \sigma_{i} \boldsymbol{v}_{i} \otimes \boldsymbol{v}_{i}, \quad \hat{\varepsilon}_{i}\left(\sigma_{1}, \sigma_{2}\right)=\frac{\partial U\left(\sigma_{1}, \sigma_{2}\right)}{\partial \sigma_{i}} .
$$

The generalization of the above to $3 \mathrm{D}$ is immediate and we omit it.

Define the elastic constants

$$
E=\frac{(\alpha-\beta)(\alpha+2 \beta)}{\alpha+\beta}, \quad \nu=\frac{\beta}{(\alpha+\beta)} .
$$

For a linear elastic isotropic solid these are Young's Modulus and Poisson's Ratio, respectively. For our 3D model, we consider uniaxial stress with principal stresses $\sigma_{i}\left(\sigma_{2}=\sigma_{3}=0\right)$ and corresponding principal strains $\varepsilon_{i}$. We find that

$$
\sigma_{1}=\left\{\begin{array}{ll}
E \varepsilon_{1}, & \varepsilon_{1} \geq 0, \\
\rho E \varepsilon_{1}, & \varepsilon_{1}<0,
\end{array} \quad \varepsilon_{2}=\varepsilon_{3}= \begin{cases}-\nu \varepsilon_{1}, & \varepsilon_{1} \geq 0 \\
-\rho \nu \varepsilon_{1}, & \varepsilon_{1}<0\end{cases}\right.
$$

In other words, there is a loss of stiffness in compression: the effective Young's Modulus (defined as the ratio of longitudinal stress and strain in uniaxial stress) is $E$ for tension and $\rho E$ for compression. The uniaxial stress-strain relation thus has the form of Fig. 1(b). At the same time, there is a weakening of the Poisson effect: the effective Poisson's Ratio-defined as minus the ratio of transverse to longitudinal strain in uniaxial stress - is equal to $\rho \nu$ in compression, less than the usual value of $\nu$ in tension. This weakening of the Poisson effect in compression is confirmed by simulations of a discrete fiber network model described in Section 6. We note that (2.19) can be written as $\sigma_{1}=Z_{\rho}\left(E \varepsilon_{1}\right), \varepsilon_{2}=\varepsilon_{3}=-Z_{\rho}\left(\nu \varepsilon_{1}\right)$ in view of (2.4). The bulk modulus also decreases by a factor of $\rho$ from hydrostatic tension to compression.

We turn to simple shear; a 2D description is sufficient. The strain tensor in simple shear has component matrix

$$
[\boldsymbol{E}]=\left(\begin{array}{cc}
0 & \gamma / 2 \\
\gamma / 2 & 0
\end{array}\right)
$$

where $\gamma$ is called the amount of shear. One notes that the principal strains change signs together with $\gamma$, (provided one maintains the order of the eigenvectors of $\boldsymbol{E}$ ). It is easy to verify that for $\gamma>0$ and $\gamma<0$ the principal strains lie in different sectors of the principal strain plane (where different quadratic branches of the energy function are in force). Accounting for this, one can compute the matrix of components of the stress tensor (in the same basis). The result is

$$
[\boldsymbol{S}]=\left(\begin{array}{cc}
N(\rho)|\gamma| & M(\rho) \gamma \\
M(\rho) \gamma & N(\rho)|\gamma|
\end{array}\right)
$$


where

$$
M(\rho)=\frac{\left(\alpha^{2}-\beta^{2}\right)[\alpha-\beta \rho+(\alpha-\beta) \rho]}{4\left(\alpha^{2}-\beta^{2} \rho\right)}, \quad N(\rho)=\frac{\alpha\left(\alpha^{2}-\beta^{2}\right)(1-\rho)}{4\left(\alpha^{2}-\beta^{2} \rho\right)} .
$$

Here $M(\rho)$ is an effective shear modulus (ratio of shear stress and amount of shear for simple shear). It is positive for any $0<\rho \leq 1$. For $\rho=1$ it equals the usual shear modulus $\mu$. We observe that the shear stress-strain relation is linear (with no discontinuities in slope), but the slope depends on the stiffness ratio $\rho$. The shear modulus $M(\rho)$ is an increasing function of $\rho$, from about $0.8 \mu$ at $\rho=0$ to the linear elastic value $\mu$ at $\rho=1$. This is plausible, since one might expect that compression weakening (lower $\rho$ ) also causes a decrease in shear stiffness.

At first glance, it seems surprising that there are normal stresses present in simple shear under small strain conditions. These occur in (2.20) provided there is compression weakening $(\rho<1)$. For $\rho=1$, the normal stresses vanish, $N(1)=0$, as expected for isotropic linear elasticity. The normal stress modulus $N(\rho)>0$ for $\rho<1$ is the ratio of normal stress to the magnitude $|\gamma|$ of the amount of shear, since in (2.20), $S_{11}=S_{22}=N(\rho)|\gamma|$. The normal stress modulus is a decreasing function of $\rho$, and vanishes at $\rho=1$. For $\rho<1$, normal stresses are positive regardless of the sign of $\gamma \neq 0$; thus to maintain the simple shear, hydrostatic tension must be applied in addition to the shear stress; this hydrostatic tension is often called "negative normal stress" in the literature [16, 17], since it corresponds to negative pressure. It corresponds to a reverse or negative Poynting effect $[18,19]$. This phenomenon has been observed and studied as a somewhat unusual characteristic of fibrous hydrogels (networks of semiflexible biopolymers) [16, 17, 8]. It is understood [17] that the underlying mechanism is loss of compression strength of fibers in the direction corresponding to the compressive principal strain in simple shear. This corresponds to compression weakening, and thus explains why our model is capable of predicting the reverse Poynting effect. For more details, see the Discussion (Section 6.)

\section{The Contracting Cell Problem in 2D}

\subsection{Formulation and Solution}

We model the situation of a contracting cell in a fibrin matrix. It turns out that radially symmetric solutions can be constructed analytically, so the cell is modelled as a disk of radius $a$ (in 2D) centered at the origin, while the matrix is the annulus $a<r<A$, where $A$ is the outside radius and $r=|\boldsymbol{x}|$ is radial distance from the center, while $\boldsymbol{x}$ is the position vector. Displacement fields with radial symmetry are of the form $\boldsymbol{u}(\boldsymbol{x})=u(r) \boldsymbol{x} / r$ in terms of the radial displacement (scalar) function $u(r)$. The principal strains and stresses are functions of $r$ :

$$
\varepsilon_{1}=\varepsilon_{r}(r)=u^{\prime}(r), \quad \varepsilon_{2}=\varepsilon_{\theta}(r)=u(r) / r
$$


where a prime indicates a derivative, while $\varepsilon_{1}$ is the radial strain and $\varepsilon_{2}$ is the circumferential strain. The equilibrium equations in terms of the radial stress $\sigma_{1}=\sigma_{r}(r)$ and hoop stress $\sigma_{2}=\sigma_{\theta}(r)$ reduce to

$$
\left(r \sigma_{r}(r)\right)^{\prime}=\sigma_{\theta}(r)
$$

We suppose that (i) the cell shrinks, and (ii) that the outside boundary of the matrix is traction free. We model (i) and (ii) by the boundary conditions

$$
u(a)=-u_{0},
$$

where $u_{0}$ is a positive constant, and

$$
\sigma_{r}(A)=0
$$

respectively. The solution of the corresponding linear elastic problem (with $\rho=1$ ) has the property that $\sigma_{r}(r)>0, \sigma_{\theta}(r)<0$ for $a<r<A$. Adopting these inequalities a priori as an ansatz in the case of the compression weakening material $(0<\rho<1)$, the stress-strain relations are given by (2.16); hence the second boundary condition (3.4) becomes

$$
\alpha u^{\prime}(A) / \rho+\beta u(A) / A=0
$$

Substituting (3.1) into (2.16), and the result into (3.2), yields a 2nd order linear ODE for $u(r)$ :

$$
r^{2} u^{\prime \prime}(r)+r u^{\prime}(r)-\rho u(r)=0 \text { for } a<r<A
$$

$u(r)$ is also subject to the boundary conditions (3.3) and (3.5). The solution of this boundary value problem is admissible provided it can be verified a posteriori that it satisfies the ansatz

$$
\sigma_{r}(r)>0, \quad \sigma_{\theta}(r)<0 \text { for } a<r<A
$$

which ensures that (2.16) holds.

The general solution of the ODE (3.6) for $u(r)$ is (letting $\xi=\sqrt{\rho}$ )

$$
u(r)=c_{1} r^{-\xi}+c_{2} r^{\xi}, \quad \xi=\sqrt{\rho} .
$$

The constants $c_{1}$ and $c_{2}$ are obtained by satisfaction of the boundary conditions (3.3) and (3.5). The result is

$$
c_{1}=-u_{0} \frac{a^{\xi} A^{2 \xi}(\alpha+\beta \xi)}{a^{2 \xi}(\alpha-\beta \xi)+A^{2 \xi}(\alpha+\beta \xi)}, \quad c_{2}=-u_{0} \frac{a^{\xi}(\alpha-\beta \xi)}{a^{2 \xi}(\alpha-\beta \xi)+A^{2 \xi}(\alpha+\beta \xi)} .
$$

The radial displacement (3.8) then takes the form

$$
u(r)=-u_{0} \frac{(\alpha+\beta \xi)\left(\frac{r}{A}\right)^{-\xi}+(\alpha-\beta \xi)\left(\frac{r}{A}\right)^{\xi}}{(\alpha+\beta \xi)\left(\frac{a}{A}\right)^{-\xi}+(\alpha-\beta \xi)\left(\frac{a}{A}\right)^{\xi}}, \quad a \leq r \leq A .
$$


The stresses are given by

$$
\begin{aligned}
& \sigma_{r}(r)=\left(u_{0} / a\right) \frac{\xi\left(\alpha^{2}-\beta^{2}\right)\left[\left(\frac{r}{A}\right)^{-\xi-1}-\left(\frac{r}{A}\right)^{\xi-1}\right]}{(\alpha+\beta \xi)\left(\frac{a}{A}\right)^{-\xi-1}+(\alpha-\beta \xi)\left(\frac{a}{A}\right)^{\xi-1}}, \\
& \sigma_{\theta}(r)=-\left(u_{0} / a\right) \frac{\xi^{2}\left(\alpha^{2}-\beta^{2}\right)\left[\left(\frac{r}{A}\right)^{-\xi-1}+\left(\frac{r}{A}\right)^{\xi-1}\right]}{(\alpha+\beta \xi)\left(\frac{a}{A}\right)^{-\xi-1}+(\alpha-\beta \xi)\left(\frac{a}{A}\right)^{\xi-1}}, \quad a \leq r \leq A .
\end{aligned}
$$

In order to verify the ansatz (3.7), we rewrite them as

$$
\begin{aligned}
& \sigma_{r}(r)=u_{0} \frac{\xi a^{\xi}\left(\alpha^{2}-\beta^{2}\right) r^{-\xi-1}\left(A^{2 \xi}-r^{2 \xi}\right)}{a^{2 \xi}(\alpha-\beta \xi)+A^{2 \xi}(\alpha+\beta \xi)}, \\
& \sigma_{\theta}(r)=-u_{0} \frac{\xi^{2} a^{\xi}\left(\alpha^{2}-\beta^{2}\right) r^{-\xi-1}\left(A^{2 \xi}+r^{2 \xi}\right)}{a^{2 \xi}(\alpha-\beta \xi)+A^{2 \xi}(\alpha+\beta \xi)}, \quad a \leq r \leq A .
\end{aligned}
$$

In view of (2.3) and since $0<\rho \leq 1(0<\xi \leq 1)$ we have $\alpha \pm \beta \xi>0$. Also $A^{2 \xi} \pm r^{2 \xi} \geq 0$ and $u_{0}>0$. It follows from the above form that the inequalities (3.7) are satisfied. Therefore, (3.10) with stresses (3.11), (3.12) provides the solution to the contracting cell problem for the constitutive law provided by the compression weakening model of Section 2.

The solution is proportional to the contractile displacement $u_{0}$ for $u_{0}>0$ (contraction). For $u_{0}<0$ (expanding cell) the ansatz (3.7) is violated. The solution for this case is obtained in Section 3.5; its implications are discussed in Section 6.

In the special case $\rho=1(\xi=1)$ we recover the linear elastic solution. The displacement is of the form $u(r)=c_{1} / r+c_{2} r$, while stresses are of the form $\sigma(r)=c_{3} / r^{2}+c_{4}$.

\subsection{Universal Bounds}

A glance at the general solution (3.8) shows that it contains a term that decays as $r$ increases but also one that increases. That raises the question whether the second term would dominate for large $r$. Recall that $a \leq r \leq A$. Because of the boundary conditions, the constants $c_{1}$ and $c_{2}$ depend on $A$; see (3.9). It turns out that $c_{2}$ decreases as $A$ increases and actually vanishes in the limit of an infinite matrix (as $A \rightarrow \infty$ ). In contrast, $c_{1}$ does not vanish in this limit. In fact, using the inequalities (2.3), $0<\xi \leq 1$, we have $\alpha \pm \beta \xi>0$ so that rewriting (3.10),

$$
\begin{gathered}
u(r)=-u_{0} \frac{A^{2 \xi}(\alpha+\beta \xi)(r / a)^{-\xi}+a^{2 \xi}(\alpha-\beta \xi)(r / a)^{\xi}}{a^{2 \xi}(\alpha-\beta \xi)+A^{2 \xi}(\alpha+\beta \xi)}, \quad a \leq r \leq A \Rightarrow \\
|u(r)| \leq u_{0} \frac{A^{2 \xi}(\alpha+\beta \xi)(r / a)^{-\xi}+a^{2 \xi}(\alpha-\beta \xi)(r / a)^{\xi}}{A^{2 \xi}(\alpha+\beta \xi)}=u_{0}(r / a)^{-\xi}+u_{0} \frac{a^{2 \xi}(\alpha-\beta \xi)(r / a)^{\xi}}{A^{2 \xi}(\alpha+\beta \xi)}
\end{gathered}
$$

But since $0<r \leq A$ we have $(a / A)^{2 \xi} \leq(a / r)^{2 \xi}$, so that the above gives the following upper bound for the displacements:

$$
|u(r)| \leq u_{0} M_{1}(r / a)^{-\xi}, \quad M_{1}=\frac{2 \alpha}{(\alpha+\beta \xi)}
$$


Since $u_{0}>0, \alpha \pm \beta \xi>0$, (3.8), (3.9) imply that each of the terms in (3.8) is negative. Also $-c_{1}>2 u_{0} a^{\xi}$ since $a<A$. This leads to the lower bound

$$
|u(r)|=-u(r) \geq 2 u_{0}(r / a)^{-\xi}
$$

Combining the last two bounds we have the following bound for the displacements induced by a circular cell of radius $a$ contracting radially with displacement $u_{0}$ in a circular matrix of arbitrary radius.

$$
u_{0} M_{1}^{-}(r / a)^{-\xi} \leq|u(r)| \leq u_{0} M_{1}^{+}(r / a)^{-\xi}, \quad M_{1}^{+}=\frac{2 \alpha}{(\alpha+\beta \xi)}, \quad M_{1}^{-}=2 .
$$

The bound is universal in the sense that it is independent of the outside radius $A$ and shows that the displacements decay with order $O\left(r^{-\sqrt{\rho}}\right)$, despite the presence of the second (growing) term in (3.8). The lower bound in (3.15) ensures that in fact $u(r)$ does not decay faster than $r^{-\sqrt{\rho}}$ (which is not guaranteed by the upper bound alone). This ensures that the decay is slower than the linear elastic one.

A similar calculation based on (3.13), (3.14) (noting for example that $A^{2 \xi}+r^{2 \xi} \leq 2 A^{2 \xi}$ ) gives universal bounds for the norm of the stress tensor $\sigma(r)=\sqrt{\sigma_{r}^{2}(r)+\sigma_{\theta}^{2}(r)}$ in the form

$$
\left(u_{0} / a\right) M_{2}^{-}\left(\frac{r}{a}\right)^{-(\sqrt{\rho}+1)} \leq|\sigma(r)| \leq\left(u_{0} / a\right) M_{2}^{+}\left(\frac{r}{a}\right)^{-(\sqrt{\rho}+1)}, M_{2}^{+}=\frac{2 \xi\left(\alpha^{2}-\beta^{2}\right)}{\alpha+\beta \xi}, M_{2}^{-}=\xi M_{2}^{+} / 4 .
$$

where the constants $M_{2}^{ \pm}$depends only on material properties $\alpha, \beta$ and $\rho=\xi^{2}$ but not on $a, A$. We conclude that stresses and displacements induced by a contracting cell in a matrix composed of compression weakening material, decay slower than in a linear elastic matrix where $u=O\left(r^{-1}\right)$ and $\sigma=O\left(r^{-2}\right)$.

\subsection{Infinite Matrix}

Taking the limit as $A \rightarrow \infty$ we obtain the displacement due to a contracting cell in an infinite matrix (with the stress approaching zero at large distances)

$$
u(r)=-u_{0}\left(\frac{r}{a}\right)^{-\xi}, \quad \xi=\sqrt{\rho}
$$

The stresses are

$$
\sigma_{r}(r)=\left(u_{0} / a\right) \frac{\xi\left(\alpha^{2}-\beta^{2}\right)}{\alpha+\beta \xi}(r / a)^{-\xi-1}, \quad \sigma_{\theta}(r)=-\xi \sigma_{r}(r), \quad a \leq r<\infty .
$$

Thus for a compression weakening material with $\rho<1(\xi<1)$ the displacements, $u(r)=O\left(r^{-\xi}\right)$, and the stresses, $\sigma(r)=O\left(r^{-\xi-1}\right)$ as $r \rightarrow \infty$. Thus both decay slower than their linear elastic counterparts, which are $O\left(r^{-1}\right)$ and $O\left(r^{-2}\right)$, respectively. The lower the compression stiffness ratio 
$\rho=\xi^{2}$, the slower the decay. We conclude that regardless of whether cell mechanosensing is based on stresses or displacements, if cells sense each other mechanically, they can do so over larger distances in fibrin networks than in materials that do not weaken in compression.

\subsection{The Case of Zero Compression Strength}

The case $\rho=0$ is interesting but tricky. The limit as $\rho \rightarrow 0$ in the solution (3.10), (3.11), (3.12) is

$$
u(r)=-u_{0}, \quad \sigma_{r}(r)=\sigma_{\theta}(r)=0, \quad a \leq r \leq A .
$$

Taking the limit as $\rho=0$ in the constitutive law (2.16), yields

$$
\sigma_{1}=\frac{\alpha^{2}-\beta^{2}}{\alpha} \varepsilon_{1}, \quad \sigma_{2}=0
$$

where $\sigma_{1}=\sigma_{r}, \varepsilon_{1}=u^{\prime}(r)$ for the radially symmetric problem. Then the equilibrium equation (3.2) becomes $\left(r \sigma_{r}(r)\right)^{\prime}=0$ or $\left(r u^{\prime}(r)\right)^{\prime}=0$. The general solution is

$$
u(r)=c_{1} \log r+c_{2}, \quad \sigma_{r}(r)=\frac{\alpha^{2}-\beta^{2}}{\alpha} \frac{c_{1}}{r} .
$$

If we enforce the boundary conditions (3.3), (3.4), the second demands $c_{1}=0$ and the first that $c_{2}=u_{0}$, thus for the bounded traction free matrix we recover (3.17). For the infinite matrix however, since the stress decays as $1 / r$, we only have (3.3) to enforce and that leaves a one parameter family of solutions

$$
u(r)=c_{1} \log (r / a)-u_{0} .
$$

If we insist though that $u(r)$ remain bounded, then necessarily $c_{1}=0$ and the only solution is (3.17).

\subsection{Expanding cells are short sighted}

We ask the following question: Suppose the cell has a choice between contracting and expanding. What is more efficient for mechanosensing in a fibrin network matrix?

To answer this in the context of our model, suppose now that we change the sign in (3.3) and require $u_{0}<0$. Then the signs in (3.11) and (3.12) are reversed, and (3.7) is violated! The previous solution with a mere sign change does not apply here. One expects that changing the sign of $u_{0}$ will reverse the signs in (3.7), so that instead of (3.7), we will make the ansatz

$$
\sigma_{r}(r)<0, \quad \sigma_{\theta}(r)>0
$$

This will put the stresses in the second, as opposed to the fourth quadrant of the principal stress plane, involving a different quadratic branch of the energy function. Then $\hat{C}$ in (2.14) will equal the 
inverse of the second, instead of the fourth matrix in (2.12). Accordingly (2.16) must be replaced by

$$
\sigma_{1}=h(\rho)\left(\alpha \varepsilon_{1}+\beta \varepsilon_{2}\right), \quad \sigma_{2}=h(\rho)\left(\beta \varepsilon_{1}+\alpha \varepsilon_{2} / \rho\right),
$$

with (3.1) still in force. This eventually results in a different ODE for $u(r)$, namely

$$
r^{2} u^{\prime \prime}(r)+r u^{\prime}(r)-\rho^{-1} u(r)=0 \text { for } a<r<A .
$$

The general solution is

$$
u(r)=c_{1} r^{-d}+c_{2} r^{d}, \quad d=1 / \sqrt{\rho} .
$$

The main difference here is that always $d>1$ and $d \rightarrow \infty$ as $\rho \rightarrow 0$. For example the solution of the cell in the infinite matrix is

$$
u(r)=-u_{0}\left(\frac{r}{a}\right)^{-d}, \quad d=1 / \sqrt{\rho},
$$

while the stresses behave as $\sigma(r)=O\left(r^{-d-1}\right)$. Thus, both displacements and stresses induced by an expanding cell decay faster than their linear elastic counterparts, which are $O\left(r^{-1}\right)$ and $O\left(r^{-2}\right)$, respectively. The lower the compression stiffness ratio $\rho=1 / d^{2}$, the faster the decay.

This result agrees with a numerical calculation in [8]: when a concentrated load is exerted in a finite element network model, the displacements decay faster (than linear elasticity predicts) in the direction towards which the force points (the "pushing" direction), and slower in the opposite, or pulling direction.

Thus cell expansion is not a good mechanism for long range mechanosensing, because displacements due to an expanding cell decay rapidly over space. In a linear elastic matrix, cell expansion and contraction produce fields with the same decay rate.

\section{The Contracting Spherical Cell in 3D}

In 3D the cell is modelled as a sphere of radius $a$ centered at the origin, while the matrix is the region $a<r<A$, or the portion of the sphere of radius $A$ outside the cell. Again, $r=|\boldsymbol{x}|$ is radial distance from the center, while $\boldsymbol{x}$ is the position vector. Displacement fields with radial symmetry are of the form $\boldsymbol{u}(\boldsymbol{x})=u(r) \boldsymbol{x} / r$ as in 2D. The principal strains and stresses are functions of $r$ :

$$
\varepsilon_{1}=\varepsilon_{r}(r)=u^{\prime}(r), \quad \varepsilon_{2}=\varepsilon_{3}=\varepsilon_{\theta}(r)=\varepsilon_{\varphi}(r)=u(r) / r,
$$

where $r, \theta, \varphi$ are spherical polar coordinates. Since $\sigma_{\theta}(r)=\sigma_{\varphi}(r)$, the equilibrium equations reduce to

$$
\left(r^{2} \sigma_{r}(r)\right)^{\prime}=2 r \sigma_{\theta}(r)
$$


We impose the same boundary conditions (3.3), (3.4) as in 2D. The solution of the corresponding linear elastic problem $(\rho=1)$ has the property that $\sigma_{r}(r)>0, \sigma_{\theta}(r)=\sigma_{\varphi}(r)<0$ for $a<r<A$. Once again we presuppose (3.7), to be verified later. Substituting (4.1) into the 3D stress-strain relations, and the result into (4.2), yields a 2nd order linear ODE for $u(r)$ :

$$
r^{2} u^{\prime \prime}(r)+2 r u^{\prime}(r)-\frac{2 \alpha \rho}{\alpha+\beta(1-\rho)} u(r)=0 \quad \text { for } a<r<A
$$

$u(r)$ is also subject to the boundary conditions (3.3) and (3.4). The solution of this boundary value problem is admissible provided it satisfies (3.7). Note that (4.3) involves the material constants $\alpha$ and $\beta$, in contrast to the ODE (3.6) for 2D. Letting

$$
g(\rho)=\frac{\alpha \rho}{\alpha+\beta(1-\rho)},
$$

the general solution of (4.3) is

$$
u(r)=c_{1} r^{\xi_{-}}+c_{2} r^{\xi_{+}}, \quad \xi_{ \pm}=\frac{1}{2}(-1 \pm \sqrt{1+8 g(\rho)}) .
$$

Observing that $g(0)=0, g(1)=1$ and that $g(\rho)$ is monotone increasing (as can be shown using (2.17)), we have that

$$
-2 \leq \xi_{-}<-1, \quad 0<\xi_{+} \leq 1
$$

Also, $\xi_{-} \rightarrow-1$ as $\rho \rightarrow 0$. Proceeding as in 2D we find the constants $c_{1}$ and $c_{2}$ in (4.4) from the boundary conditions (3.3) and (3.4). The solution can be written as follows. Define the constants

$$
P_{ \pm}= \pm\left([\alpha+\beta(1-\rho)] \xi_{ \pm}+2 \beta \rho\right), \quad Q_{ \pm}=\alpha+\beta\left(1+\xi_{ \pm}\right), \quad R=\frac{(\alpha-\beta)(\alpha+2 \beta)}{(\alpha+\beta)^{2}-\beta(\alpha+3 \beta) \rho}
$$

The displacement is

$$
u(r)=-u_{0} \frac{P_{+}\left(\frac{r}{A}\right)^{\xi_{-}}+P_{-}\left(\frac{r}{A}\right)^{\xi_{+}}}{P_{+}\left(\frac{a}{A}\right)^{\xi_{-}}+P_{-}\left(\frac{a}{A}\right)^{\xi_{+}}}
$$

The stresses are

$$
\begin{aligned}
& \sigma_{r}(r)=\left(u_{0} / a\right) \frac{R P_{+} P_{-}\left[\left(\frac{r}{A}\right)^{\xi_{--1}}-\left(\frac{r}{A}\right)^{\xi_{+}-1}\right]}{P_{+}\left(\frac{a}{A}\right)^{\xi_{-}-1}+P_{-}\left(\frac{a}{A}\right)^{\xi_{+}-1}}, \\
& \sigma_{\theta}(r)=-\left(u_{0} / a\right) \frac{\rho R\left[Q_{-} P_{+}\left(\frac{r}{A}\right)^{\xi_{-}-1}+Q_{+} P_{-}\left(\frac{r}{A}\right)^{\xi_{+}-1}\right]}{P_{+}\left(\frac{a}{A}\right)^{\xi_{-}-1}+P_{-}\left(\frac{a}{A}\right)^{\xi_{+}-1}}, \quad a \leq r \leq A .
\end{aligned}
$$

Using (2.17), (4.4) and (4.5), one can show that

$$
P_{ \pm}>0, \quad Q_{ \pm}>0, \quad R>0
$$


This implies that the inequalities (3.7) are satisfied and the solution is admissible. It is possible to derive global bounds (as for the 2D solution) of the form

$$
\begin{gathered}
u_{0} M_{1}^{-}\left(\frac{r}{a}\right)^{\xi_{-}} \leq|u(r)| \leq u_{0} M_{1}^{+}\left(\frac{r}{a}\right)^{\xi_{-}}, \\
\left(u_{0} / a\right) M_{2}^{-}\left(\frac{r}{a}\right)^{\xi_{-}-1} \leq|\sigma(r)| \leq\left(u_{0} / a\right) M_{2}^{+}\left(\frac{r}{a}\right)^{\xi_{-}-1} .
\end{gathered}
$$

where $\xi_{-}$is the negative root in (4.4), (4.5), while $M_{1}^{ \pm}$and $M_{2}^{ \pm}$are constants that depend only on $\alpha, \beta$ and $\rho$, that is, on material constants only, but not on the geometry (not on $A, a$ ). For $\rho<1$, in view of (4.5) we have $\xi_{-}>-2$, hence these bounds imply that the decay is always slower than the linear elastic case $\rho=1$ (for which $\xi_{-}=-2, \xi_{+}=1$ ).

In the limiting case $\rho=0$, the $3 \mathrm{D}$ constitutive law reduces to

$$
\sigma_{i}=\left\{\begin{array}{ll}
E \varepsilon_{i}, & \varepsilon_{i} \geq 0, \\
0, & \varepsilon_{i}<0,
\end{array}, \quad i=1,2,3\right.
$$

For the contracting inclusion, this means that the only nonzero stress component is $\sigma_{r}(r)=E u^{\prime}(r)$ since the hoop stains are compressive, and the equilibrium equation (4.2) reduces to $\left(r^{2} \sigma(r)\right)^{\prime}=0$. The implies that $s(r)=c_{1} / r^{2}$, hence $u(r)=c_{2} / r+c_{3}$ for constants $c_{i}$. The solution to the contraction inclusion in an infinite matrix is $u(r)=-u_{0} a / r$. This validates a heuristic argument made by Notbohm et al. (see the discussion involving Eq. (1) in [9]).

\section{Results}

To test the hypothesis that displacements in a compression weakening elastic material propagate over a longer range than in a linear elastic one, we develop a homogeneous continuum model, but one with a nonlinear elastic constitutive law, in which the principal stresses depend on the principal strains in a special, piecewise linear fashion. Following Notbohm et al. [9], we choose the stiffness in compression to be lower than that in tension. In 1D this is easy to do; such a stress-strain law that weakens in compression is shown in Fig. 1(b). The slope (stiffness) is less in compression that in tension. The ratio of the two is a parameter $\rho$ in the range

$$
0<\rho \leq 1
$$

We call this parameter the compression stiffness ratio. When $\rho=1$ we are back to linear elasticity.

It is rather challenging to construct higher dimensional constitutive models that weaken in compression in an acceptable fashion. The stresses have to be continuous functions of the strains (though 
not necessarily differentiable), and they must be derivable from a strain energy density function, otherwise the elastic constitutive law is thermodynamically unsound. This task is the subject of Section 2. The result is a strain energy density function that is a piecewise quadratic function of the principal strains. Its level curves in the principal strain plane are shown in Fig. 2(a), while those of the underlying linear elastic one are shown in Fig. 2(b).

Once the $2 \mathrm{D}$ constitutive model is constructed, it is more straightforward to generalize it to a $3 \mathrm{D}$ constitutive law in Section 2.2.

The mechanical behavior of the constitutive model is described in Section 2.3. A comparison with certain types of unusual experimental and simulated behavior characteristic of fibrin can be found in Section 6.

In Section 3 we consider a problem intended to model the contracting cell in a matrix that exhibits loss of stiffness in compression. The matrix may be finite but possibly large compared to the cell; we do account for external boundaries. We start with 2D. The elastostatic problem for our constitutive model is tractable in the radially symmetric case. In Section 3.1, the cell is modelled as a contracting circle of radius $a$; the matrix as a disk of radius $A$, with the cell at its center. The case $A>>a$ is typical, although we leave $A$ and $a$ arbitrary $(A>a>0)$. The matrix external boundary $(r=A)$ is free of applied forces (traction-free), while the cell boundary ( $r=a$ ) suffers a prescribed negative radial displacement $-u_{0}$. This gives two boundary conditions. The matrix is assumed to be composed of the material with constitutive law developed in Section 2. This is characterized by two elastic constants and the stiffness ratio $\rho$.

We obtain the solution to this problem in Section 3.1. The displacement field is radial, of the form

$$
u(r)=c_{1} r^{-\sqrt{\rho}}+c_{2} r^{\sqrt{\rho}}, \quad a<r<A,
$$

so that the first term in (5.1) decays, while the second grows, as the distance $r$ from the origin increases. The relevant stress components take the general form

$$
\sigma_{i}(r)=c_{3} r^{-(\sqrt{\rho}+1)}+c_{4} r^{\sqrt{\rho}-1}, \quad a<r<A,
$$

where $\sigma_{i}$ stands for either $\sigma_{r}$ or $\sigma_{\theta}$. The constants $c_{1}$ through $c_{4}$ are determined by the boundary conditions. For the complete closed-form solution see (3.10)-(3.12). When we set $\rho=1$ we recover the linear elastic solution $u(r)=c_{1} / r+c_{2} r$. For $0<\rho<1$, the decreasing term does decay slower than the corresponding linear elastic term, but the role of the growing terms seems unclear. We deal with this in Section 3.2. We observe that the constants $c_{2}, c_{4}$ tend to zero in the limit as $A \rightarrow \infty$. This suggests that the growing terms may remain small. We find that this is indeed the case and deduce 
bounds for the displacement and the norm $\sigma(r)$ of the stress tensor in the form

$$
\begin{gathered}
u_{0} M_{1}^{-}\left(\frac{r}{a}\right)^{-\sqrt{\rho}} \leq|u(r)| \leq u_{0} M_{1}^{+}\left(\frac{r}{a}\right)^{-\sqrt{\rho}}, \\
\left(u_{0} / a\right) M_{2}^{-}\left(\frac{r}{a}\right)^{-(\sqrt{\rho}+1)} \leq|\sigma(r)| \leq\left(u_{0} / a\right) M_{2}^{+}\left(\frac{r}{a}\right)^{-(\sqrt{\rho}+1)} .
\end{gathered}
$$

These inequalities involve only the negative exponent $-\sqrt{\rho}$, although they bound the entire displacement and stress in (5.1), (5.2), including the growing terms. Also, they are universal, in the sense that the constants $M_{1}^{ \pm}$and $M_{2}^{ \pm}$are independent of the size of the matrix $A$ and the inclusion radius $a$ (the geometry); see (3.15), (3.16). Rather, they only depend on the elastic constants and the stiffness ratio $\rho$. Also the dependence of the bounds on $r$ is through $r / a$, or distance measured in cell radii.

The lower bounds in (5.3) prove that the displacement and stress fields exterior to a contracting spherical inclusion in a compression weakening material (governed by the constitutive model developed here) decay slower that the corresponding linear elastic ones, which satisfy analogous bounds, obtained by replacing $\sqrt{\rho}$ by 1 in (5.3)

The continuum solution (5.1) is in good quantitative agreement with numerical simulations of the fiber discrete network model of Notbohm et al. [9]. See Section 6 and Table 1 for details.

The case of the infinite matrix is briefly dealt with in Section 3.3. In Section 3.4 we also consider the case $\rho=0$, which is a singular limit.

We consider hypothetical cells that expand instead of contracting in Section 3.5. Surprisingly, the solution for a compression weakening material decays faster than the linear elastic one. This explains why expansion, as opposed to contraction, of individual cells is not conducive to long-range mechanosensing. See Section 6 for the implications of this.

The 3D contracting cell problem is formulated in Section 4 as that of a sphere of radius $a$, contracting with radial displacement $-u_{0}$ at the center of a spherical matrix of radius $A$, whose external boundary is traction free. The 3D constitutive law of Section 2.2 is used. The solution (4.6)-(4.8) is more complicated than the $2 \mathrm{D}$ one, but qualitatively very similar. The solution has the form

$$
u(r)=c_{1} r^{\xi_{-}}+c_{2} r^{\xi_{+}},
$$

but the exponents $\xi_{ \pm}$now depend on the elastic moduli, in addition to the compression stiffness ratio $\rho$. The exponents $\xi_{ \pm}$satisfy

$$
-2 \leq \xi_{-}<-1, \quad 0<\xi_{+} \leq 1
$$

while universal bounds of the form (4.9) are valid. Once again, in 3D the decay is slower than in a linear elastic matrix where the displacement would decay with $\xi_{-}=-2$. Fits of displacement data from the 3D fiber network model simulations of Notbohm et al. [9] to the form $u=\mathrm{Cr}^{-n}$ 
for $\rho=0.1$ give an exponent $n=0.67$ for a high 3D connectivity of 14 , and $n=0.82$ for a low connectivity of 3.5 , close to the value considered representative of fibrin, compared to the continuum model prediction of $\xi_{-}=-1.1$. Experiments involving fibroblasts in 3D fibrin [9] give $n=0.52$. The agreement is not as good as in $2 \mathrm{D}$, but the qualitative conclusions remain the same.

\section{Discussion}

\subsection{A Continuum Model for Fibrin}

In Section 2 we construct a new hyperelastic constitutive model for compression-weakening materials. We view this constitutive law as a continuum model for a fibrin network, and possibly other fibrous materials. The essential characteristic we wish to capture is loss of stiffness in compression. In a discrete fibrous network, this happens because individual fibers buckle under compression. In our continuum model, it is embodied in a special constitutive nonlinearity. Specifically, each principal stress is a piecewise linear function of the principal strains in a way that generalizes the behavior of Figure 1(b) (a 1D idealization of buckling behavior) to 2D and 3D.

We compare the behavior of the model under certain homogeneous deformations described in Section 2.3 with experimental observations for fibrin.

The uniaxial stress-strain relation (2.19) predicted by our model is linear in tension, and neglects the gradual nonlinear strain stiffening characteristic of many polymeric fibrous materials under tension, including fibrin [15, 20, 21]. In some cases however, fibrin exhibits very nearly linear behavior in uniaxial tension up to strains well beyond the scope of our small-strain model [22, 23].

Choosing piecewise linear relations for our model has certain advantages. First, it allows us to solve analytically some model problems that provide insight into mechanosensing by contractile cells. Second, it is the simplest model that accounts for compression weakening without introducing other nonlinearities. This minimalist approach allows us to isolate and study the effect of compression weakening on the slow decay of elastic fields due to contracting inclusions. We find such an effect in the absence of stiffening, as discussed below in Section 6.2.

One prediction of our model constitutive law is a weakening of the Poisson effect in uniaxial compression. The effective Poisson's ratio (minus the ratio of transverse to longitudinal strain in uniaxial stress) is equal to $\nu_{c}=\rho \nu_{t}$ in compression, compared to the value of $\nu_{t}$ in tension; see (2.19). We are not aware of experiments reporting both tensile and compressive values of the effective Poisson ratio for fibrin. We compare this prediction with simulations of a discrete fiber network model $[8,9]$, where each fiber has a stress-strain curve as in Fig. 1(b), with stiffness ratio $\rho$. For 2D uniaxial stress, simulation results for the ratio of the compressive and tensile values of the effective 
Poisson ratio are within $3 \%$ to the fit $\nu_{c} / \nu_{t}=0.74 \rho$ for the values tested $(\rho=0.1,0.3,0.5,0.7$ ). The fiber network model undergoes weakening of the Poisson effect in compression, in qualitative agreement with the prediction of our model, $\nu_{c} / \nu_{t}=\rho$, in that the ratio is less than one and increases linearly with $\rho$.

Fibrous polymer networks exhibit unusual behavior under homogeneous simple shear: they develop normal stresses corresponding to a negative hydrostatic pressure, with a sign opposite to that of the usual Poynting effect in nonlinear elasticity of rubberlike solids [18, 19]. This reverse Poynting effect is often termed "negative normal stresses." 3 The phenomenon was experimentally observed in fibrin [16] and simulated using discrete fiber network models [17]. The underlying mechanism was identified in [17] as "compressive buckling of the individual filaments". Consistently with this, our model predicts the presence of such normal stresses corresponding to a negative pressure, whenever the compression stiffness ratio $\rho$ is less than unity, namely, in the presence of compression weakening; see (2.20). Instead, when $\rho=1$ and the model reduces to linear elasticity, normal stresses vanish in simple shear.

The network model of [17] predicts a normal stress that is quadratic for small strains, but becomes proportional to the absolute value of the shear stress for large strains. Our piecewise smooth constitutive law predicts that normal stresses in simple shear, and normal strains under pure shear stress, are each proportional to the absolute value of the shear stress. This result is very similar to Fig. 4 of [17] (for small bending stiffness) and to the experimental data shown in Fig. 4(b) of [16]. For pure shear stress, our results agree with the network model simulation results of [9] (supplemental Fig. S6) in that the ratio of normal strain to the absolute value of shear strain is constant, negative, and increases in magnitude as the the stiffness ratio $\rho$ decreases from 1 to 0 .

Another unusual type of behavior experimentally observed in fibrin [22] and exhibited by the network model of $[8,9]$ is termed "negative compressibility", referring to a decrease of volume during uniaxial tension. This only occurs at large strains above $10 \%$ in both experiments and simulations $[22,8,9]$. This is not a surprise, since such behavior in the small strain regime for an isotropic material would require a negative bulk modulus, which is inconsistent with the positive definiteness of the elasticity tensor in linearized elasticity. Our model behaves as an ordinary linear elastic isotropic solid during uniaxial tension, and does not exhibit such an effect.

These remarks suggest that negative compressibility is not the primary factor in the observed slow decay of displacements due to contractile cells in fibrin, as the latter phenomenon does not seem to require large deformations [8]. As we discuss next, the model supports the conclusions of Notbohm et al. [9] that the primary cause is compression weakening.

\footnotetext{
${ }^{3}$ Presumably this term refers to the sign of the pressure, rather than that of the normal stress components, which are tensile, hence positive according the the usual sign convention.
} 


\subsection{Spatial Decay of Elastic Fields Due to Contractile Cells}

In Section 3 we model a contractile cell in a fibrin matrix as a contracting circular inclusion embedded in a material governed by our model constitutive law in 2D. This is the continuum analog of the 2D contracting inclusion problem for a finite element model of a fiber network [8,9], with individual fibers having a force-elongation relation as in Fig. 1(b) (where the ratio $\rho$ of slopes in compression and tension is chosen between 0 and 1 ). The solution (5.1), (5.2) to the continuum contracting inclusion problem, together with the bounds (5.3), clearly predicts that both displacements and stresses decay slower with distance from a contractile inclusion in a material that weakens in compression than in a linear elastic one.

Notbohm et al. [9] performed simulations of the contracting inclusion using a finite element model of a fiber network with each node acting as a hinge for the elements terminating at it, randomness in nodal positions, and different values of the connectivity (the average number of fibers meeting at a node). Each fiber has a stress-strain curve as in Fig. 1(b), with stiffness ratio $\rho$. The numerical radial displacement data were fit to the form

$$
u(r)=C_{1} r^{-n}+C_{2} r^{n}
$$

with fitting parameters $C_{1}, C_{2}, n$. This form is consistent with the continuum solution (5.1) of the present work. The value $\rho=0.1$ was used in the simulations [9]. The present continuum model also involves $\rho$; the corresponding solution (5.1) predicts that $n=\sqrt{\rho}$. The fit for $n$ resulting from the network model data depends on the connectivity (the average number of fibers meeting at a node) of the network simulated. The network with the highest connectivity of 8 is likely to behave closest to the continuum model. In this case fits of the numerical data give $n=0.36$. Our continuum solution (5.1) for the choice $\rho=0.1$ yields the prediction $n=0.32$. For $\rho=1$ our model reduces to linear elasticity and does not weaken in compression. The discrete network model fit yields $n=0.89$ for $\rho=1$.

Additional simulations were performed using a network of connectivity 8 and no randomness in nodal positions, for three different values of $\rho$ and for both a contracting and an expanding inclusion, for the purpose of comparing the network and continuum models. Data were fit to the form (6.1) and also to

$$
u(r)=C_{1} r^{-p_{1}}+C_{2} r^{p_{2}}
$$

with fitting parameters $C_{1}, C_{2}, p_{1}$ and $p_{2}$. The continuum model predicts $p_{1}=p_{2}=n=\sqrt{\rho}$ for the contracting inclusion, and $p_{1}=p_{2}=n=1 / \sqrt{\rho}$ for the expanding one; see Section 3.5, eq. (3.21). The agreement is quite satisfactory. The results of the comparison are summarized in Table 1. 
(a)

\begin{tabular}{|c|c|c|c|c|c|c|c|c|c|}
\hline$\rho$ & $\sqrt{\rho}$ & $n$ & $p_{1}$ & $p_{2}$ & $\rho$ & $1 / \sqrt{\rho}$ & $n$ & $p_{1}$ & $p_{2}$ \\
\hline$\overline{c 0.3}$ & 0.547 & 0.532 & 0.531 & 0.543 & 0.3 & 1.83 & $\bar{~} 1.87$ & 1.87 & 1.83 \\
\hline 0.1 & 0.316 & 0.337 & 0.340 & 0.320 & 0.1 & 3.16 & 3.16 & 3.17 & 3.16 \\
\hline 0.03 & 0.173 & 0.226 & 0.235 & 0.176 & 0.03 & 5.77 & 5.17 & 5.17 & 5.17 \\
\hline
\end{tabular}

Table 1: (a) Comparison of the theoretical prediction $\sqrt{\rho}$ for the decay power (from the solution (5.1) of the continuum model) to the power $n$ from fits of (6.1), and to $p_{1}$ and $p_{2}$ from fits of (6.2) to simulation data using the discrete fiber model of [9], for the contracting inclusion, for three values of $\rho$. (b) Analogous comparison for the expanding inclusion; the theoretical prediction is $1 / \sqrt{\rho}$.

When compression weakening is suppressed ( $\rho=1$ in both models) the decay rate of numerical solutions is comparable to the linear elastic one, while the continuum problem reduces to the linear elastic one.

According to the present model, a contracting inclusion of radius $a$ in an infinite matrix in 2D with stress approaching zero at infinity induces the displacement field

$$
u(r)=-u_{0}\left(\frac{r}{a}\right)^{-\sqrt{\rho}}, \quad a<r<\infty,
$$

while the stress field is proportional to $r^{-(1+\sqrt{\rho})}$. Thus the slow decay rate in the presence of compression weakening $(\rho<1)$ has unbounded range. The solution does not approach the 2D linear elastic one, $u(r)=-u_{0}(r / a)^{-1}$, as $r \rightarrow \infty$.

To solve the contracting inclusion problem, previous studies [24, 25] have used different nonlinear models that do not include compression weakening, but exhibit strain stiffening at large tensile strains in different forms. Wang et al. [25] consider tension-driven alignment of fibers as the mechanism responsible for long-range force transmission in fibrous matrices. In their constitutive model, they account for this by means of a tension-stiffening term. This nonlinearity would eventually cause higher stiffness in tension than compression, provided the cell stretches the matrix far enough into the nonlinear regime to cause an appreciable difference in tensile and compression stiffness. For fibrin, this could mean large tensile strains induced by the cell, exceeding the stiffening threshold of about $10 \%$ [21, 22, 23], probably at a high energetic cost, to facilitate mechanosensing. Moreover, as strains decay with distance from the cell, the difference between tension and compression stiffness would become negligible in a tension-stiffening model, and the displacement would approach the linear elastic decay rate. Thus the slow decay of elastic fields would be confined to a "nonlinear zone" around the cell. The larger the critical strain for stiffening, the smaller the size of such a nonlinear zone.

In contrast, microbuckling involves a sudden drop of stiffness in compression, which for fibrin occurs at a very small strain of about $4 \times 10^{-4}[15,9]$, two orders of magnitude below the strains 
required for tension stiffening. While both compression weakening and tension stiffening occur in fibrin, and contracting cells induce both compressive (hoop) and tensile (radial) strains, compression weakening is likely to be the dominant mechanism, since it is engaged at much lower cell-induced strain levels. In the experiments of $[8,9]$ slow displacement decay was observed at cell-induced strains of $2 \%$, below the level required for appreciable stiffening $[21,22,23]$ and less than the high levels required for prediction of long range transmission of forces by the model of [25].

Our model treats the buckling strain as negligible. Hence, its behavior is not smooth at zero strain, and does not approach linear elasticity for small strains. As a result, long-range propagation of elastic fields occurs without spatial limit, and for arbitrarily small cell-applied contractile displacement $u_{0}$. In practice, the cell contractile strain $u_{0} / a$ must exceed a small buckling strain, and the nonlinear zone of slow decay would be large but finite. In experiments [8,9], a transition to a linear elastic spatial decay rate was not observed within the measurement range of $100 \mu \mathrm{m}$, of the order of 10 cell radii.

Other simulations of model networks without compression weakening, but with gradual stiffening in tension and continuous slope at zero strain, predicted displacement decay rates close to the linear elastic one, even for large cell strain $u_{0} / a \approx 10 \%[8,9]$. These results and also the experiments of [11], suggest that strain stiffening is not the primary factor causing the observed slow displacement decay.

We conclude that microbuckling in fibrous materials, modelled as compression weakening in the continuum setting, is directly responsible for long-range propagation of elastic fields induced by cell contraction, thus it facilitates mechanosensing in fibrous biopolymer matrices.

Why do cells contract instead of expanding to facilitate mechanosensing? Probably a good answer to this question is: because they can! That is, it may only be possible for the cell to exert tension on the matrix, because the mechanism is essentially "winches pulling on ropes", i.e., myosin-II motors pulling on actin filaments. So it may be physically difficult for the cell to generate large forces by pushing on the matrix while it is stationary. Until recently, pushing forces applied by single cells within a fibrous matrix had not been observed [26]. Pushing forces only seem to occur during invasive cellular migration into the matrix; even then, pushing may result as a reaction force balancing the contraction of cellular protrusions [26, 27]. Immobile cells contract during the mechanosensing process, before any growth into the matrix occurs $[4,9]$.

What could have driven cells to evolve so as to exhibit almost entirely contractile behavior while stationary? To understand this, we consider a related question in Section 3.5: Suppose a stationary cell has a choice between contracting and expanding. Which is more efficient for mechanosensing in a fibrin network matrix? Because of nonlinearity, specifically the constitutive asymmetry between 
tension and compression, reversing the sign of the applied boundary displacement $-u_{0}$ does not simply multiply the solution by -1 , as would happen in linear elasticity. Instead, if the cell expands and pushes at the matrix (let $u_{0}<0$ ) the solution (3.21) is still of the form (5.1), (5.2) but with different exponents $\pm 1 / \sqrt{\rho}$ in place of $\pm \sqrt{\rho}$. Bounds similar to (5.3) still hold, but with negative exponent $-1 / \sqrt{\rho}$ that approaches $-\infty$ as $\rho \rightarrow 0$. As a result, the displacements and stresses due to an expanding cell in a compression weakening material decay faster than in a linear elastic material. Thus expanding cells would cloak themselves from other cells; this is counterproductive for mechanosensing. We conclude that contractile behavior of individual cells in a fibrous, compression weakening matrix, is far more more efficient for long-range mechanosensing than expansion. It seems that cells have evolved accordingly.

\section{Acknowledgments}

This work was motivated by experiments performed in collaboration with Dr. Ayelet Lesman and Professor David Tirrell under a grant from the National Science Foundation (Division of Materials Research No. 0520565) through the Center for the Science and Engineering of Materials at the California Institute of Technology. P.R. acknowledges the hospitality of the Graduate Aerospace Laboratories at the California Institute of Technology (GALCIT).

\section{References}

[1] Dennis E Discher, Paul Janmey, and Yu-li Wang. Tissue cells feel and respond to the stiffness of their substrate. Science, 310(5751):1139-1143, 2005.

[2] Chun-Min Lo, Hong-Bei Wang, Micah Dembo, and Yu-li Wang. Cell movement is guided by the rigidity of the substrate. Biophysical Journal, 79(1):144-152, 2000.

[3] Cynthia A Reinhart-King, Micah Dembo, and Daniel A Hammer. Cell-cell mechanical communication through compliant substrates. Biophysical Journal, 95(12):6044-6051, 2008.

[4] Jessamine P Winer, Shaina Oake, and Paul A Janmey. Non-linear elasticity of extracellular matrices enables contractile cells to communicate local position and orientation. PLoS One, 4(7):e6382, 2009.

[5] Quanming Shi, Rajarshi P Ghosh, Hanna Engelke, Chris H Rycroft, Luke Cassereau, James A Sethian, Valerie M Weaver, and Jan T Liphardt. Rapid disorganization of mechanically interacting systems of mammary acini. Proceedings of the National Academy of Sciences, 111(2):658$663,2014$. 
[6] Shijie He, Yewang Su, Baohua Ji, and Huajian Gao. Some basic questions on mechanosensing in cell-substrate interaction. Journal of the Mechanics and Physics of Solids, 70:116-135, 2014.

[7] Viola Vogel and Michael Sheetz. Local force and geometry sensing regulate cell functions. Nature Reviews Molecular Cell Biology, 7(4):265-275, 2006.

[8] Jacob Notbohm. Dynamics of Cell-Matrix Mechanical Interactions in Three Dimensions. $\mathrm{PhD}$ thesis, California Institute of Technology, 2013.

[9] Jacob Notbohm, Ayelet Lesman, Phoebus Rosakis, David A Tirrell, and Guruswami Ravichandran. Microbuckling of fibrin provides a mechanism for cell mechanosensing. Journal of The Royal Society Interface, 12(108):20150320, 2015.

[10] C Franck, S Hong, SA Maskarinec, DA Tirrell, and G Ravichandran. Three-dimensional fullfield measurements of large deformations in soft materials using confocal microscopy and digital volume correlation. Experimental Mechanics, 47(3):427-438, 2007.

[11] Mathilda S Rudnicki, Heather A Cirka, Maziar Aghvami, Edward A Sander, Qi Wen, and Kristen L Billiar. Nonlinear strain stiffening is not sufficient to explain how far cells can feel on fibrous protein gels. Biophysical Journal, 105(1):11-20, 2013.

[12] R Lakes, P Rosakis, and A Ruina. Microbuckling instability in elastomeric cellular solids. Journal of Materials Science, 28(17):4667-4672, 1993.

[13] Oleg V. Kim, Rustem I. Litvinov, John W. Weisel, and Mark S. Alber. Structural basis for the nonlinear mechanics of fibrin networks under compression. Biomaterials, 35(25):6739 - 6749, 2014.

[14] Oleg V Kim, Xiaojun Liang, Rustem I Litvinov, John W Weisel, Mark S Alber, and Prashant K Purohit. Foam-like compression behavior of fibrin networks. Biomechanics and modeling in mechanobiology, pages 1-16, 2015.

[15] Izabela K Piechocka, Rommel G Bacabac, Max Potters, Fred C MacKintosh, and Gijsje H Koenderink. Structural hierarchy governs fibrin gel mechanics. Biophysical journal, 98(10):2281-2289, 2010.

[16] Paul A Janmey, Margaret E McCormick, Sebastian Rammensee, Jennifer L Leight, Penelope C Georges, and Fred C MacKintosh. Negative normal stress in semiflexible biopolymer gels. Nature Materials, 6(1):48-51, 2007. 
[17] Enrico Conti and Fred C MacKintosh. Cross-linked networks of stiff filaments exhibit negative normal stress. Physical review letters, 102(8):088102, 2009.

[18] JH Poynting. On pressure perpendicular to the shear planes in finite pure shears, and on the lengthening of loaded wires when twisted. Proceedings of the Royal Society of London. Series A, Containing Papers of a Mathematical and Physical Character, 82(557):546-559, 1909.

[19] L Angela Mihai and Alain Goriely. Positive or negative poynting effect? the role of adscititious inequalities in hyperelastic materials. Proceedings of the Royal Society A: Mathematical, Physical and Engineering Science, 467(2136):3633-3646, 2011.

[20] Cornelis Storm, Jennifer J Pastore, Fred C MacKintosh, Tom C Lubensky, and Paul A Janmey. Nonlinear elasticity in biological gels. Nature, 435(7039):191-194, 2005.

[21] Blayne A Roeder, Klod Kokini, Jennifer E Sturgis, J Paul Robinson, and Sherry L VoytikHarbin. Tensile mechanical properties of three-dimensional type i collagen extracellular matrices with varied microstructure. Journal of biomechanical engineering, 124(2):214-222, 2002.

[22] A.E.X. Brown, R.I. Litvinov, D.E. Discher, P.K. Purohit, and J.W. Weisel. Multiscale mechanics of fibrin polymer: Gel stretching with protein unfolding and loss of water. Science, 325(5941):741-744, 2009.

[23] Albert James Licup, Stefan Münster, Abhinav Sharma, Michael Sheinman, Louise M Jawerth, Ben Fabry, David A Weitz, and Fred C MacKintosh. Stress controls the mechanics of collagen networks. arXiv preprint arXiv:1503.00924, 2015.

[24] Yair Shokef and Samuel A Safran. Scaling laws for the response of nonlinear elastic media with implications for cell mechanics. Physical Review Letters, 108(17):178103, 2012.

[25] Hailong Wang, AS Abhilash, Christopher S Chen, Rebecca G Wells, and Vivek B Shenoy. Long-range force transmission in fibrous matrices enabled by tension-driven alignment of fibers. Biophysical journal, 107(11):2592-2603, 2014.

[26] Jacob Notbohm, Ayelet Lesman, David A Tirrell, and Guruswami Ravichandran. Quantifying cell-induced matrix deformation in three dimensions based on imaging matrix fibers. Integrative Biology, 2015.

[27] Katarina Wolf and Peter Friedl. Extracellular matrix determinants of proteolytic and nonproteolytic cell migration. Trends in cell biology, 21(12):736-744, 2011. 